\title{
Flat connections, the Alexander invariant, and Casson's invariant
}

\author{
Christopher M. Herald
}

This paper explores the relationship between the flat moduli space of a homology knot complement and other topological invariants of the knot. We define an invariant by counting the flat orbits with trace of the meridinal holonomy fixed and give a formula for the invariant in terms of the TristamLevine equivariant knot signature and the Casson invariant of the homology 3 -sphere in which the knot lives.

Casson defined a topological invariant, now known as the Casson invariant, for closed oriented homology 3-spheres which, roughly speaking, counts (one half) the number of irreducible representations from the fundamental group of the 3-manifold into $S U(2)$ modulo conjugation (see [AM]). Shortly thereafter, Taubes provided an analytic definition of the same invariant, counting instead flat $S U(2)$ connections modulo gauge equivalence (see $[\mathrm{T}]$ ). These papers, along with related ones by Walker [Wa] and Floer $[\mathrm{F}]$, have inspired a long list of papers both from the topological viewpoint and from the gauge theoretic one.

In 1992, Lin defined an $S^{3}$ knot invariant by counting the number of trace-free irreducible representations of the knot group into $S U(2)$ modulo conjugation (see $[\mathrm{Li}]$ ). Here trace-free means that all knot meridians are taken to trace-free matrices. He then showed by a clever topological argument that this invariant equals one half of the knot signature. Ruberman suggested a generalization of this result involving equivariant knot signature which removed the trace-free condition. For the case of 2-bridge knots, a similar formula was conjectured by Heusener in [He].

The aim of this article is to establish a general formula for arbitrary knots in homology 3 -spheres. In the special case of knots in $S^{3}$, this proves the formula suggested by Ruberman. Our main result states that if $\kappa: S^{1} \rightarrow X$ is a smooth knot in an oriented homology 3 -sphere then (under certain transversality assumptions) the number of conjugacy classes of nonabelian representations $\rho: \pi_{1}(X \backslash \kappa) \rightarrow S U(2)$ taking the meridians to matrices of trace $2 \cos \alpha$ equals minus four times the Casson invariant of $X$ minus one 
half the Tristam-Levine knot signature $\operatorname{Sign} B_{\kappa}\left(e^{i 2 \alpha}\right)$. The conjugacy classes are counted with signs corresponding to oriented intersection numbers.

The techniques used in this paper are gauge theoretic rather than topological. In particular, we consider flat $S U(2)$ connections modulo gauge equivalence, rather than fundamental group representations to $S U(2)$ modulo conjugation. The association taking each flat connection to its holonomy representation gives an identification of the flat moduli space with the space of representations modulo conjugation.

The general picture is as follows. Let $Y$ be the complement of an open tubular neighborhood of a smooth knot $\kappa$ in an oriented homology sphere $X$. Let $\mathcal{M}$ denote the space of flat connections on $Y$ modulo gauge equivalence, and let $\mathcal{M}^{*}$ denote the subset consisting of equivalence classes of irreducible connections. My work in [H1] and [H2] shows that after a small perturbation of the flatness equation, if necessary, $\mathcal{M}^{*}$ is a smooth 1-manifold, compact except for open ends which limit to interior points in the abelian moduli space $\mathcal{M} \backslash \mathcal{M}^{*}$, which is an arc.

There is a natural restriction map $r$ from $\mathcal{M}$ to $\mathcal{M}_{T^{2}}$, the flat moduli space for the boundary torus. This moduli space is the "pillowcase" orbifold $T^{2} / \mathrm{Z}_{2}$, which is topologically a 2 -sphere. Equivalence classes of irreducible flat connections on $Y$ whose holonomy representations take knot meridians to matrices of trace $2 \cos \alpha$ correspond to points in $r^{-1}\left(S_{\alpha}\right)$ for a certain circle $S_{\alpha} \subset \mathcal{M}_{T^{2}}$. Thus the points to be counted are intersections between the immersion $r: \mathcal{M}^{*} \rightarrow \mathcal{M}_{T^{2}}$ and the circle $S_{\alpha}$.

The signs of the intersections are determined by considering the double cover $\tilde{\mathcal{M}}^{*}$ of $\mathcal{M}^{*}$. In the second section we describe a method by which $\tilde{\mathcal{M}}^{*}$ is given a natural orientation, once one is chosen on $H^{1}(Y ; \mathbf{R})$. There is a corresponding restriction map $\tilde{r}: \tilde{\mathcal{M}} \rightarrow \tilde{\mathcal{M}}_{T^{2}}=T^{2}$. The intersection points to be counted correspond in a one-to-one fashion with $\tilde{r}^{-1}\left(\tilde{S}_{\alpha}\right) \cap \tilde{\mathcal{M}}^{*}$, where $\tilde{S}_{\alpha}$ is one component in the double cover of $S_{\alpha}$. An orientation on $\tilde{S}_{\alpha}$ is also determined by the ones on $H^{1}(Y ; \mathbf{R})$. The last piece of information we need is an orientation on $\tilde{\mathcal{M}}_{T^{2}}$. Its tangent space is canonically isomorphic to $H^{1}(\partial Y ; \mathbf{R})$ and so receives an orientation from Poincaré duality on $\partial Y$, oriented as the boundary of $Y \subset X$. We adopt the convention that an oriented basis for $T_{x} \partial Y$ followed by an outward normal vector gives an oriented basis for $T_{x} Y$.

Several more pieces of notation are necessary to state our main theorem. Choose a Seifert surface $F$ for $\kappa$ and fix an oriented normal vector field on $F$. Choose a basis of curves $\left\{x_{i}\right\}_{1 \leq i \leq 2 g}$ for $H_{1}(F ; \mathbf{R})$ and let $x_{i}^{+}$denote the pushoff of $x_{i}$ in the positive normal direction. We then define the Seifert pairing $V$ to be the matrix with entries $V_{i j}=\operatorname{link}\left(x_{i}, x_{j}^{+}\right)$. We use this to define, for 
each unit complex number $t$, a matrix $B_{\kappa}(t)=(1-t) V+\left(1-t^{-1}\right) V^{T}$. The signature of this Hermitian matrix is called the Tristam-Levine signature of the knot and is a function from $U(1)$ to $\mathbf{Z}$. The matrix $B_{\kappa}(t)$ is related to the symmetrized Alexander matrix $A_{\kappa}(t)=t^{\frac{1}{2}} V-t^{-\frac{1}{2}} V^{T}$ by

$$
\left(t^{-\frac{1}{2}}-t^{\frac{1}{2}}\right) A_{\kappa}(t)=B_{\kappa}(t),
$$

and the Alexander polynomial of the knot is

$$
\Delta_{\kappa}(t)=\operatorname{det}\left(V^{T}-t V\right)=\left(1-t^{-1}\right)^{-2 g} \operatorname{det}\left(B_{\kappa}(t)\right) .
$$

Our main theorem is the following, where $\lambda(X)$ denotes the Casson invariant of $X$ (see $[\mathrm{AM}]$ and $[\mathrm{T}]$ ).

Theorem 0.1. For any $0<\alpha<\pi$ with $\Delta_{\kappa}\left(e^{i 2 \alpha}\right) \neq 0$, if $\mathcal{M}$ is nondegenerate, then

$$
\tilde{r}\left(\tilde{\mathcal{M}}^{*}\right) \cdot \tilde{S}_{\alpha}=-4 \lambda(X)-\frac{1}{2} \operatorname{Sign} B_{k}\left(e^{i 2 \alpha}\right) .
$$

If $\mathcal{M}$ is degenerate, then after a small perturbation of the flatness equation this formula holds.

Remark: The intersection number on the left side of the formula is the number of flat orbits in $\mathcal{M}_{h}^{*}$ with $\operatorname{tr}\left(\operatorname{hol}_{\mu}(A)\right)=2 \cos \alpha$, counted with signs. We only state the formula in terms of the double cover because doing so allows us to interpret the correct signs as oriented intersection numbers. We will also see that the Casson invariant has an interpretation as an oriented intersection number, namely, $-\frac{1}{4} \tilde{r}\left(\tilde{\mathcal{M}}^{*}\right) \cdot \tilde{S_{0}}$. This collection of intersections corresponds to the double cover of the set of orbits of connections on $Y$ which may be extended to flat connections on $X$.

As a special case, when $\kappa$ is a knot in $S^{3}$, we get the generalization of Lin's theorem suggested by Ruberman.

Corollary 0.2. Let $Y$ be the complement of a knot $\kappa$ in $S^{3}$. Then for any $0<\alpha<\pi$ with $B_{\kappa}\left(e^{i 2 \alpha}\right)$ invertible, if $\mathcal{M}$ is nondegenerate, $\tilde{r}\left(\tilde{\mathcal{M}}^{*}\right) \cdot \tilde{S}_{\alpha}=$ $-\frac{1}{2} \operatorname{Sign} B_{\kappa}\left(e^{i 2 \alpha}\right)$. If $\mathcal{M}$ is degenerate, then after a small perturbation this formula holds.

We have learned from David Austin that he has independently found a proof of a result similar to Corollary 3 , but his proof is more along the lines of Akbulut and McCarthy's treatment of the Casson invariant (see [AM]). Acknowledgements: I would like to express my gratitude to my thesis advisor Tom Mrowka, who first suggested this problem to me and with 
whom I have had many long and fruitful discussions on the subject of this paper. I have also benefitted greatly from discussions with Hans Boden, Paul Kirk, Eric Klassen, Andy Nicas, and Ron Stern.

\section{Background and Notation.}

In this section we review the basic gauge theory setup and the generic structure of the flat (or perturbed flat) moduli space for a knot complement. This material is described in full detail in [H1] and [H2].

Let $\kappa$ be a smooth knot in an oriented homology sphere $X$ with open tubular neighborhood $\nu(\kappa)$. Set $Y=X \backslash \nu(\kappa)$, with the orientation inherited from $X$. We fix a Riemannian metric on $Y$, and let $*: \Omega^{p}(Y ; s u(2)) \rightarrow$ $\Omega^{3-p}(Y ; s u(2))$ be the Hodge star operator.

We consider the trivial $S U(2)$ bundle $P=Y \times S U(2)$. By fixing a trivialization of $P$ we obtain an identification of the space of $S U(2)$ connections on $Y$ with $\Omega^{1}(Y ; s u(2))$, the space of 1 -forms on $Y$ with $s u(2)$ values. Let $\mathcal{A}$ denote the completion of this space with respect to the $L_{2}^{2}$ Soboloev norm. The gauge group in this context is $\mathcal{G}=\operatorname{Map}(Y, S U(2))$, completed with the $L_{3}^{2}$ norm. $\mathcal{A}_{T^{2}}$, and $\mathcal{G}_{T^{2}}$ are defined similarly, using the $L_{\frac{3}{2}}^{2}$ and $L_{\frac{5}{2}}^{2}$ norms, respectively. In this paper we will not be explicit about Sobolev norms.

$S U(2)$ connections may be divided into classes according to the size of their stabilizer, which is isomorphic to $\mathbf{Z}_{2}, U(1)$, or $S U(2)$. Such connections are called irreducible, abelian, and central, respectively. (Note that according to this definition, abelian and central are mutually exclusive.) It is useful to divide $\mathcal{A}$ into $\mathcal{A}=\mathcal{A}^{*} \cup \mathcal{A}^{U(1)}$ where $\mathcal{A}^{*}$ denotes the set of irreducible connections and $\mathcal{A}^{U(1)}$ denotes the set of reducible (that is, abelian or central) connections. Similarly we divide the space of gauge equivalence classes $\mathcal{B}=\mathcal{A} / \mathcal{G}$ into $\mathcal{B}=\mathcal{B}^{*} \cup \mathcal{B}^{U(1)}$.

To each connection $A$ is associated its curvature 2 -form, $F(A)=d A+$ $A \wedge A \in \Omega^{2}(Y ; s u(2))$, and the connection $A$ is said to be flat if its curvature is zero. The flat moduli space $\mathcal{M}=\{A \in \mathcal{A} \mid F(A)=0\} / \mathcal{G}$ of $Y$ is the object of primary interest in this paper. It has a topological interpretation as $\left.\operatorname{Hom}\left(\pi_{1}(Y), S U(2)\right) / S U(2)\right)$, the set of fundamental group representations modulo conjugation.

The flat moduli space of a knot complement is in general a very singular space and hence is difficult to work with (although interesting results have been proven using the its real algebraic structure, for example in [He]). For this reason, we define a class of admissible perturbations of the flatness equation as follows. 
Let $\left\{\gamma_{i}: S^{1} \times D^{2} \rightarrow Y\right\}_{1 \leq i \leq n}$ be a collection of embeddings of the solid torus into $Y$ whose images are disjoint. Let $\eta$ be the product of a nonnegative bump function on $D^{2}$ with support in the interior and the standard volume form on $D^{2}$. Let $\left\{\bar{h}_{i}: \mathbf{R} \rightarrow \mathbf{R}\right\}_{1 \leq i \leq n}$ be a collection of $C^{2}$ functions. Let $\operatorname{tr} \operatorname{hol}_{\gamma_{i}}(x, A)$ be the trace of the holonomy of the connection $A$ around the curve $\gamma_{i}\left(S^{1} \times\{x\}\right)$. We define a function $h: \mathcal{A} \rightarrow \mathbf{R}$ by the formula

$$
h(A)=\sum_{i=1}^{n} \int_{D^{2}} \bar{h}_{i}\left(\operatorname{tr} \operatorname{hol}_{\gamma_{i}}(x, A)\right) \eta(x) .
$$

Definition 1. A function $h$ constructed in this way is called an admissible perturbation function.

Given an admissible perturbation $h: \mathcal{A} \rightarrow \mathbf{R}$, we define a function $\zeta_{h}(A): \mathcal{A} \rightarrow \Omega^{1}(Y ; s u(2))$ by

$$
\zeta_{h}(A)=-\frac{1}{2 \pi} * F(A)+\nabla h(A)
$$

Definition 2. We say that a connection $A$ is perturbed flat (with respect to a perturbation $h$ ) if it satisfies the equation $\zeta_{h}(A)=0$. The perturbed flat moduli space is the quotient $\mathcal{M}_{h}=\zeta_{h}^{-1}(0) / \mathcal{G}$. We also decompose this into the set of irreducible orbits $\mathcal{M}_{h}^{*}=\mathcal{M}_{h} \cap \mathcal{B}^{*}$ and the reducible orbits $\mathcal{M}_{h}^{U(1)}=\mathcal{M}_{h} \cap \mathcal{B}^{U(1)}$.

One point should be made about the notation. The flat moduli space of $Y$ or its strata will generally be denoted by $\mathcal{M}_{h}, \mathcal{M}_{h}^{*}$, and $\mathcal{M}_{h}^{U(1)}$, with no further adornment. We will denote the corresponding moduli spaces of a 3manifold $M$ which is different from $Y$ by $\mathcal{M}_{h}(M), \mathcal{M}_{h}^{*}(M)$, and $\mathcal{M}_{h}^{U(1)}(M)$.

We will denote the flat moduli space for the torus by $\mathcal{M}_{T^{2}}$. The holonomy representation of any flat connection on $T^{2}$ is abelian, so its image lies in a maximal torus $U(1) \subset S U(2)$. Any such maximal torus in $S U(2)$ can be conjugated to itself with the reverse orientation. Thus,

$$
\mathcal{M}_{T^{2}}=\operatorname{Hom}\left(\pi_{1}\left(T^{2}\right), S U(2)\right) / A d=\operatorname{Hom}(\mathbf{Z} \oplus \mathbf{Z}, U(1)) / \mathbf{Z}_{2}=T^{2} / \mathbf{Z}_{2} .
$$

This flat moduli space is topologically a 2 -sphere with four distinguished points, namely the fixed points of the involution (the central orbits).

Given a connection $A$ which is perturbed flat with respect to an admissible perturbation $h$, we will define associated absolute and relative cohomology groups, denoted by $\mathcal{H}_{A, h}^{1}(Y ; s u(2))$ and $\mathcal{H}_{A, h}^{1}(Y, \partial Y ; s u(2))$, in Section 
3. Roughly, they arise as the kernel and cokernel of the linearization of the perturbed flatness equation. When $h=0$, these correspond to cohomology with coefficients in the twisted $s u(2)$ bundle of the flat connection.

Definition 3. We will say that $\mathcal{M}_{h}$ is nondegenerate if it satisfies the following 5 properties (and otherwise degenerate):

(a) The only orbits in $\mathcal{M}_{h}$ which are central when restricted to $\partial Y$ are central on $Y$.

(b) For each central connection $A, \mathcal{H}_{A, h}^{1}(Y, \partial Y ; s u(2))=0$.

(c) For finitely many noncentral orbits $[A] \in \mathcal{M}_{h}^{U(1)}, \operatorname{dim} \mathcal{H}_{A, h}^{1}(Y, \partial Y ; s u(2))=$ 2 and $\operatorname{Stab} A$ acts nontrivially on $\mathcal{H}_{A, h}^{1}(Y, \partial Y ; s u(2))$. For all remaining noncentral abelian orbits $\operatorname{dim} \mathcal{H}_{A, h}^{1}(Y, \partial Y ; s u(2))=0$.

(d) At each abelian orbit where there is nontrivial relative first cohomology the family of symmetric matrices $H_{t}$ (defined below) has transverse spectral flow.

(e) For each $[A] \in \mathcal{M}_{h}^{*}, \operatorname{dim} \mathcal{H}_{A, h}^{1}(Y, \partial Y ; s u(2))=1$.

Conditions (a) and (b) clearly hold for $h=0$, and they continue to hold for small $h$. Condition (c) insures that $\mathcal{M}_{h}^{U(1)}$ is a smooth 1-manifold, even at the points where the cohomology jumps. In fact, for $h$ small, $\mathcal{M}_{h}^{U(1)}$ consists of a single component connecting the two central orbits.

To define the matrix $H_{t}$ in condition (d) we first choose a family of connections $A_{t}$ whose orbits parametrize an open set in $\mathcal{M}_{h}^{U(1)}$ and with $\operatorname{dim}\left(\mathcal{H}_{A_{0}, h}^{1}(Y, \partial Y ; s u(2))\right)=2$. Let $V$ denote the orthogonal complement of $T_{\left[A_{0}\right]} \mathcal{M}_{h}^{U(1)}$ in $\mathcal{H}_{A_{0}, h}^{1}(Y ; s u(2))$. Then we define a 1-parameter family of bilinear forms on $V$ by the formula

$$
H_{t}(\alpha, \beta)=\left\langle * d_{A_{t}, h} \alpha, \beta\right\rangle .
$$

$H_{t}$ is symmetric and $\operatorname{Stab}\left(A_{0}\right)=U(1)$ invariant.

When $h=0$, a jump in $\mathcal{H}_{A}^{1}(Y ; s u(2))$ occurs (for abelian connections) at connections where, up to gauge, $\operatorname{hol}_{\mu} A=\exp (\mathbf{i} \theta)$ and $\operatorname{hol}_{\lambda} A=1$ where $B_{\kappa}\left(e^{i 2 \theta}\right)$ is degenerate.

The structure of $\mathcal{M}_{h}$ was described in [H1]. It is summarized in the next theorem. Let $\overline{\mathcal{M}_{h}^{*}}$ denote the closure of $\mathcal{M}_{h}^{*}$ in $\mathcal{M}_{h}$. 
Theorem 1.1. (Theorem 15 and Corollary 24, [H1]; also, see Theorem 12, [H2]) For generic admissible perturbations $h, \overline{\mathcal{M}_{h}^{*}}$ and $\mathcal{M}_{h}^{U(1)}$ are compact smooth 1-manifolds. $\partial \overline{\mathcal{M}_{h}^{*}}$ consists of orbits in the interior of $\mathcal{M}_{h}^{U(1)}$. $\partial \mathcal{M}_{h}^{U(1)}$ consists of exactly two orbits, the central orbits. Furthermore, $r: \mathcal{M}_{h} \rightarrow \mathcal{M}_{T^{2}}$ is an immersion on each stratum taking only the central orbits of $\mathcal{M}_{h}$ to the central orbits of $\mathcal{M}_{T^{2}}$

Definition 4. The orbits in $\overline{\mathcal{M}_{h}^{*}} \cap \mathcal{M}_{h}^{U(1)}$ are called bifurcation points.

Remark: The proof of Theorem 1.1 amounts to showing that the set of perturbations for which $\mathcal{M}_{h}$ is degenerate has codimension 1 , and then using local implicit function theorem arguments to show that these cohomological conditions guarantee that $\mathcal{M}_{h}$ has the structure in the theorem.

\section{The Double Cover of the Moduli Space.}

In this section we will discuss the double cover of the moduli space (without the central orbits). This double cover has the same type of bifurcation point singularities where the different strata meet, but we will see that its 1-dimensional strata receive natural orientations from an orientation on $H^{1}(Y)$ or, equivalently, a choice of generator $[\mu] \in H_{1}(Y ; \mathbf{Z})$.

We begin by fixing a maximal torus in $\{\exp (\mathbf{i} \theta)\} \hookrightarrow S U(2)$. Here we identify $s u(2)$ with the space of purely imaginary quaternions, with standard basis elements $\mathbf{i}, \mathbf{j}$, and $\mathbf{k}$. We will abuse the notation and refer to this maximal torus as $U(1)$. The adjoint representation of $U(1)$ on $s u(2)$ reduces to a trivial representation on $\operatorname{span}\{\mathbf{i}\}$, which we will identify with $\mathbf{R}$, and a weight two representation on $\operatorname{span}\{\mathbf{j}, \mathbf{k}\}$, which we will identify with $\mathbf{C}$.

First note that by considering flat connections on the torus with holonomy values in $U(1) \subset S U(2)$ we obtain a natural double cover $\tilde{\mathcal{M}}_{T^{2}}$ of $\mathcal{M}_{T^{2}}$, branched at the central points. Specifically, $\tilde{\mathcal{M}}_{T^{2}}$ is the space of flat $u(1)$ valued connection 1 -forms divided by the group of $U(1)$ valued gauge transformations.

We define

$$
\hat{\mathcal{A}}=\left\{A \in \mathcal{A}|A|_{\partial Y} \in \Omega^{1}(\partial Y ; \mathbf{R})\right\}
$$

and

$$
\hat{\mathcal{G}}=\left\{g \in \mathcal{G}|g|_{\partial Y} \in \operatorname{Map}(\partial Y, U(1))\right\} .
$$

Let $d_{A, h}=\frac{1}{2 \pi} d_{A}-* \operatorname{Hessh}(A)$. Then $-* d_{A, h}$ is the linearization of $\zeta_{h}$. Define

$$
\Omega_{\tau}^{p}(Y ; s u(2))=\left\{a \in \Omega^{p}(Y ; s u(2))|a|_{\partial Y}=0\right\}
$$


and

$$
\Omega_{\nu}^{p}(Y ; s u(2))=\left\{a \in \Omega^{p}(Y ; s u(2))|* a|_{\partial Y}=0\right\} .
$$

Let $U$ be a contractible family of admissible perturbation functions.

We consider the family of elliptic operators

$$
K_{h}(A): \Omega_{\tau}^{0}(Y ; s u(2)) \oplus \Omega_{\nu}^{1}(Y ; s u(2)) \rightarrow \Omega^{0}(Y ; s u(2)) \oplus \Omega^{1}(Y ; s u(2)),
$$

parametrized by $(A, h) \in \hat{\mathcal{A}} \times U$, given by

$$
K_{h}(A)(\sigma, \tau)=\left(d_{A}^{*} \tau, d_{A} \sigma+* d_{A, h} \tau\right) .
$$

If $\zeta_{h}(A)=0$, then

$$
\operatorname{ker} K_{h}(A)=\mathcal{H}_{A}^{0}(Y, \partial Y ; s u(2)) \oplus \mathcal{H}_{A, h}^{1}(Y ; s u(2))
$$

and

$$
\operatorname{coker} K_{h}(A)=\mathcal{H}_{A}^{0}(Y ; s u(2)) \oplus \mathcal{H}_{A, h}^{1}(Y, \partial Y ; s u(2)) .
$$

Here $\mathcal{H}_{A}^{0}(Y ; s u(2)), \mathcal{H}_{A, h}^{1}(Y ; s u(2)), \mathcal{H}_{A, h}^{1}(Y ; \partial Y ; s u(2))$, and $\mathcal{H}_{A}^{0}(Y, \partial Y ; s u(2))$ are the harmonic spaces representing the cohomology groups of the Fredholm elliptic complex

$$
\Omega^{0} \stackrel{d_{A}}{\rightarrow} \Omega^{1} \stackrel{* d_{A, h}}{\rightarrow} \Omega^{1} \stackrel{d_{A}^{*}}{\rightarrow} \Omega^{0} .
$$

The last cohomology group is always trivial when $h=0$, these cohomology groups correspond to the cohomology with coefficients in the twisted flat $s u(2)$ bundle determined by the flat connection.

Notice that if $A \in \hat{\mathcal{A}}$ is a perturbed flat connection and $\left.A\right|_{\partial Y}$ is noncentral then all the harmonic forms have boundary values in $u(1)$. The virtual bundle Ind $K_{h}=\operatorname{ker} K_{h}-\operatorname{coker} K_{h}$ is orientable since $\hat{\mathcal{A}}$ is an affine space. We will refer to an orientation on $\operatorname{Ind} K_{h}(A)$ by specifying one on $\operatorname{ker} K_{h}(A) \oplus$ $\operatorname{coker} K_{h}(A)$.

Lemma 2.1. (Lemma 53 of [H1])

The action of $\hat{\mathcal{G}}$ on the index bundle Ind $K_{h}$ is orientation preserving.

For the remainder of this section, let $h$ be a fixed nondegenerate admissible perturbation function. For any noncentral $A \in \zeta_{h}^{-1}(0) \cap \hat{\mathcal{A}}$,

$$
\mathcal{H}_{A}^{0}(\partial Y ; s u(2))=T_{\mathrm{id}} \operatorname{Stab}\left(\left.A\right|_{\partial Y}\right) \cong u(1),
$$

which is canonically oriented. The kernel ker $K_{h}(A)$ contains the tangent space to the stratum $\left(\tilde{\mathcal{M}}_{h}^{*}\right.$ or $\left.\tilde{\mathcal{M}}_{h}^{U(1)}\right)$ containing $[A]$. If $[A]$ is a bifurcation 
point, however, the kernel contains more. In the latter case, a space complementary to the tangent space of the stratum receives a canonical orientation from $\mathcal{H}_{A}^{0}(\partial Y ; s u(2))$. Furthermore, $\operatorname{coker} K_{h}(A)$ (which always has positive dimension) also receives a canonical orientation from $\mathcal{H}_{A}^{0}(\partial Y ; s u(2))$. This can be seen as follows. There are several cases to consider.

If $[A] \in \tilde{\mathcal{M}}_{h}^{U(1)}$ is not a bifurcation point, then the restriction map $\mathcal{H}_{A}^{0}(Y ; s u(2)) \rightarrow \mathcal{H}_{A}^{0}(\partial Y ; s u(2))$ is an isomorphism and $\mathcal{H}_{A, h}^{1}(Y, \partial Y ; s u(2))=0$. If $[A] \in \tilde{\mathcal{M}}_{h}^{*}$ then $\mathcal{H}_{A}^{0}(Y ; s u(2))=0$ and $\mathcal{H}^{1}(Y ; s u(2))$ and $\mathcal{H}^{1}(Y ; s u(2))$ are both 1-dimensional. In this case, the coboundary map in the long exact sequence of the pair $(Y, \partial Y)$ gives a canonical isomorphism from $\mathcal{H}_{A}^{0}(Y ; s u(2))$ to $\mathcal{H}_{A, h}^{1}(Y, \partial Y ; s u(2))$.

If $[A] \in \tilde{\mathcal{M}}_{h}^{U(1)}$ is a bifurcation point, then the restriction map $\mathcal{H}_{A}^{0}(Y ; s u(2)) \rightarrow \mathcal{H}_{A}^{0}(\partial Y ; s u(2))$ is injective, but $\mathcal{H}_{A, h}^{1}(Y, \partial Y ; s u(2))$ and the kernel of the restriction map $r: \mathcal{H}_{A, h}^{1}(Y ; s u(2)) \rightarrow \mathcal{H}_{A}^{1}(\partial Y ; s u(2))$ are both real 2-dimensional, with $\operatorname{Stab}(A)$ acting with weight 2 to give a complex structure on each. If $[A] \in \tilde{\mathcal{M}}_{h}^{*}$ then $\mathcal{H}_{A}^{0}(Y ; s u(2))=0$ and $\mathcal{H}^{1}(Y ; s u(2))$ and $\mathcal{H}^{1}(Y ; s u(2))$ are both 1-dimensional. In this case, the coboundary map in the long exact sequence of the pair $(Y, \partial Y)$ gives a canonical isomorphism from $\mathcal{H}_{A}^{0}(Y ; s u(2))$ to $\mathcal{H}_{A, h}^{1}(Y, \partial Y ; s u(2))$.

It follows that an orientation on the virtual index bundle determines one on the 1-dimensional strata of $\tilde{\mathcal{M}}_{h}$ for each nondegenerate $h$. We fix an orientation on this index bundle by specifying it on one fiber; namely, we orient ker $K_{0}(\Theta) \oplus \operatorname{coker} K_{0}(\Theta)$, where $\Theta$ denotes the trivial connection.

Our orientation will depend on an orientation of $H^{1}(Y ; \mathbf{R})$ or, equivalently, a choice of primitive generator $[\mu] \in H^{1}(Y ; \mathbf{Z})$. Let $\mu^{*}$ denote the harmonic 1-form on $Y$ with Neumann boundary conditions and satisfying $\int_{\mu} \mu^{*}=1$. Then we take the orientation on $\operatorname{ker} K_{0}(\Theta) \oplus \operatorname{coker} K_{0}(\Theta)$ determined by the basis

$$
\left\{\mu^{*} \otimes \mathbf{i}, \mu^{*} \otimes \mathbf{j}, \mu^{*} \otimes \mathbf{k}, \mathbf{i}, \mathbf{j}, \mathbf{k}\right\} .
$$

We end this section by noting that the $\mathbf{Z}_{2}$ action on the double cover does not preserve this orientation on $\tilde{\mathcal{M}}_{h}$.

\section{Perturbing.}

In order to obtain nondegenerate moduli spaces in general, it is necessary to perturb the flatness equations. In this section we describe the admissible perturbation functions and prove that it is possible to find small perturbations such that the moduli spaces satisfy certain technical conditions that 
will be needed in Lemma 5.1.

The standard class of admissible perturbation functions $h$ in this context (see, for example, $[\mathrm{T}],[\mathrm{F}],[\mathrm{H} 1]$ ) is defined as follows. Let $x$ be a pair of coordinates on the disk $D^{2}$. Let $\eta(x)$ be a radially symmetric bump function on $D^{2}$ which vanishes near the boundary and let $d x$ be the standard measure on $D^{2}$. Given a finite collection of disjoint embeddings $\left\{\gamma_{i}: S^{1} \times D^{2} \rightarrow\right.$ $Y\}_{i=1, \ldots, n}$ and a collection of $C^{2}$ adjoint invariant functions $\left\{h_{i}: S U(2) \rightarrow\right.$ $\mathbf{R}\}_{i=1, \ldots, n}$, we define a gauge invariant function on $\mathcal{A}$ given by

$$
h(A)=\sum_{i=1}^{n} \int_{D^{2}} h_{i}\left(\operatorname{hol}_{\gamma_{i}}(x, A)\right) \eta(x) d x .
$$

Functions constructed in this way are said to be admissible.

For our purposes it will be sufficient to fix a single collection of thickened loops $\left\{\gamma_{i}\right\}_{i=1, \ldots, n}$. The collection is required to be sufficiently large in the sense of [H1], Section 6, Theorem 15. We allow ourselves to vary the functions $h_{i}$ to achieve nondegeneracy, transversality, etc. Let $\mathcal{H}=\left[C_{S U(2)}^{2}(S U(2), \mathbf{R})\right]^{n}$, the product of $n$ copies of the space of Adinvariant $C^{2}$ functions from $S U(2)$ to $\mathbf{R}$.

Theorem 3.1. Fix $0<\alpha<\pi$ with $\Delta_{\kappa}\left(e^{2 i \alpha}\right) \neq 0$. Then there exist arbitrarily small perturbations $h \in \mathcal{H}$ for which $\mathcal{M}_{h}$ satisfies (1)-(5) of Lemma 5.1 .

Proof. By the proof of Theorem 15 in [H1] there is a neighborhood $V \subset \mathcal{H}$ of 0 such that the set $V_{0} \subset V$ of perturbations for which $\mathcal{M}_{h}$ is degenerate has codimension $\geq 1$. Furthermore, Proposition 34 in [H1] implies that $V$ may be chosen small enough that the set $V_{1} \subset V$ of perturbations for which $r\left(\mathcal{M}_{h}^{*}\right)$ does not intersect $S_{\alpha}$ transversely also has codimension 1 .

Choose a path $h_{t}:(-1,1) \rightarrow V$, with $h_{0}=0$, which meets $V_{0}$ and $V_{1}$ transversely. This will ensure that there is an $\epsilon>0$ such that $\mathcal{M}_{h_{t}}$ satisfies conditions (1) and (2) for each $0<t<\epsilon$.

To meet condition (4), note that for $t$ small enough, the map $\operatorname{tr}\left(\operatorname{hol}_{\mu}\right): \mathcal{M}_{h_{t}}^{U(1)} \rightarrow[-2,2]$ given by $[A] \mapsto \operatorname{tr}\left(\operatorname{hol}_{\mu}(A)\right)$ is a diffeomorphism on the interior and continuous on the whole space. By another ( $t$-dependent) perturbation using a curve near $\partial Y$ parallel to $\mu, r\left(\mu_{h_{t}}^{U(1)}\right)$ can be realigned with the unperturbed abelian stratum. See Lemma 61 and Example 1, p. 64 , of [H1], for details. 
That condition (3) holds for $t$ sufficiently small may be seen as follows. Suppose $\tilde{S}_{\alpha}$ intersects $\tilde{r}\left(\tilde{\mathcal{M}}_{h_{t}}^{U(1)}\right)$ at a bifurcation point for arbitrarily small $t>0$. Then there are Fredholm operators arbitrarily close to $K_{A^{\alpha}}(Y)$ (namely $K_{A^{\alpha}, h_{t}}(Y)$ ) with larger kernel than $\operatorname{ker} K_{A^{\alpha}}(Y)$. This contradicts the fact that $\operatorname{dim} \operatorname{ker} K$ is an upper semi-continuous function on the space of Fredholm operators from one separable Hilbert space to another.

The path $h_{t}, 0 \leq t \leq \frac{\epsilon}{2}$, constructed in this manner, automatically satisfies condition (5).

\section{The Local Picture Near a Bifurcation Point.}

In this section we fix a primitive $[\mu] \in H_{1}(Y ; \mathbf{Z})$ to specify an orientation on the 1-dimensional strata of $\tilde{\mathcal{M}}_{h}$ as in the previous section. We assume furthermore that $\mathcal{M}_{h}$ is nondegenerate and fix $A_{0} \in \zeta_{h}^{-1}(0) \cap \hat{\mathcal{A}}$ such that $\left[A_{0}\right]$ is a bifurcation point on the $U(1)$-stratum. For concreteness say that $\operatorname{hol}_{\mu}\left(A_{0}\right)=\exp (\sigma \mathbf{i})$ where $\sigma \in(0, \pi)$ (thereby specifying in which half of the double cover of the moduli space $\left[A_{0}\right]$ lies). We will explore the Kuranishi picture for the moduli space near $\left[A_{0}\right]$.

Let $\Pi_{0}$ denote the orthogonal projection

$$
\Pi_{0}: \Omega^{0}(Y ; s u(2)) \oplus \Omega^{1}(Y ; s u(2)) \rightarrow \mathcal{H}_{A_{0}}^{0}(Y ; s u(2)) \oplus \mathcal{H}_{A_{0}, h}^{1}(Y, \partial Y ; s u(2)) .
$$

We shall obtain a description of the restriction

$$
\Pi_{0} \circ K_{h}(A): \mathcal{H}_{A_{0}, h}^{1}(Y ; s u(2)) \rightarrow \mathcal{H}_{A_{0}}^{0}(Y ; s u(2)) \oplus \mathcal{H}_{A_{0}, h}^{1}(Y, \partial Y ; s u(2)),
$$

for $A$ near $A_{0}$. This will enable us to sort out the orientations induced on the irreducible and abelian strata near $\left[A_{0}\right]$ from the one on $\mathcal{H}_{A}^{0}(\partial Y ; s u(2))$, in terms of the direction of the spectral flow of $H_{t}$ at $t=0$.

We begin by studying a part of this operator, namely, its restriction to the orthogonal complement in $\mathcal{H}_{A_{0}, h}^{1}(Y ; s u(2))$ of the $U(1)$ stratum tangent space. As pointed out above, $\operatorname{Stab}\left(A_{0}\right)$ is naturally identified with the fixed (oriented) $U(1) \subset S U(2)$. The action of $\operatorname{Stab}\left(A_{0}\right)$ on $\Omega^{1}(Y ; s u(2))$ gives a natural complex structure on this orthogonal complement, denoted by $\mathcal{H}_{A_{0}, h}^{1}(Y ; \mathbf{C})$, and on $\mathcal{H}_{A_{0}, h}^{1}(Y, \partial Y ; s u(2))$. We denote both of these complex structures by $J$.

Let $\vec{u} \in \mathcal{H}_{A_{0}}^{0}(Y ; s u(2))=T_{\mathrm{id}} \operatorname{Stab}\left(A_{0}\right)$ point in the positive direction. Recall that the map $j: \mathcal{H}_{A_{0}, h}^{1}(Y, \partial Y ; s u(2)) \rightarrow \mathcal{H}_{A_{0}, h}^{1}(Y ; s u(2))$ in the long exact sequence of the pair is in fact $L^{2}$ orthogonal projection between the 
harmonic spaces representing the cohomology groups. Choose nonzero cohomology classes $\vec{a}, \vec{b}$, and $\vec{c}$, as follows. Choose $\vec{c} \in \mathcal{H}_{A_{0}, h}^{1}(Y, \partial Y ; \mathbf{C})$ and let $\vec{b}=j \vec{c} \in \mathcal{H}_{A_{0}, h}^{1}(Y ; \mathbf{C})$. Note that $\vec{b}$ and $\vec{c}$ differ by an exact form. Let $\vec{a} \in \mathcal{H}_{A_{0}}^{1}(Y ; \mathbf{R})$ be a vector such that the orientation coming from the basis $\{\vec{a}, \vec{b}, J \vec{b}, \vec{u}, \vec{c}, J \vec{c}\}$ agrees with the orientation on $\operatorname{ker} K_{h}\left(A_{0}\right) \oplus \operatorname{coker} K_{h}\left(A_{0}\right)=$ $\mathcal{H}_{A_{0}, h}^{1}(Y ; s u(2)) \oplus \mathcal{H}_{A_{0}}^{0}(Y ; s u(2)) \oplus \mathcal{H}_{A_{0}, h}^{1}(Y, \partial Y ; s u(2))$.

Let $A_{t}$ be a 1-parameter family of connections through $A_{0}$ such that $\left[A_{t}\right]$ gives a smooth parameterization of $\mathcal{M}_{h}^{U(1)}$ We further require that the parameterization be chosen so that $\left.\frac{d}{d t} A_{t}\right|_{t=0}=\vec{a}$. Define $H_{t}$ to be the cohomological pairing $H_{t}\left(z, z^{\prime}\right)=\left\langle * d_{A_{t}, h} z, z^{\prime}\right\rangle$ on the orthogonal complement of $T_{\left[A_{0}\right]} \mathcal{M}_{h}^{U(1)}$ in $\mathcal{H}_{A_{0}, h}^{1}(Y ; s u(2))$.

Proposition 4.1. The pairing has the form $H_{t}=\lambda(t)$ Id for some real valued function $\lambda(t)$ with $\lambda(0)=0$ and $\lambda^{\prime}(0) \neq 0$.

Proof. $H_{t}: \mathbf{C} \rightarrow \mathbf{C}$ is a $U(1)$ equivariant map and hence has the form $\lambda(t) e^{i \theta(t)}$ for some real valued functions $\lambda$ and $\theta$. We first show that $H_{t}$ is symmetric, which shows that $\theta(t)=0$.

The Hessian of $h$ is symmetric. To see that $* d_{A_{t}}$ is also symmetric, let $z$ and $z^{\prime}$ be harmonic forms in $\mathcal{H}_{A_{0}}^{1}(Y ; \mathbf{C})$. These forms are necessarily exact when restricted to $\partial Y$; say, $\left.z\right|_{\partial Y}=d_{A_{0}} \xi$ and $\left.z^{\prime}\right|_{\partial Y}=d_{A_{0}} \xi^{\prime}$. Then,

$$
\begin{aligned}
\left\langle * d_{A_{t}} z, z^{\prime}\right\rangle-\left\langle * d_{A_{t}} z^{\prime}, z\right\rangle & =-\int_{Y} \operatorname{tr}\left(d_{A_{t}} z \wedge z^{\prime}-z \wedge d_{A_{t}} z^{\prime}\right) \\
& =-\int_{\partial Y} \operatorname{tr}\left(z \wedge z^{\prime}\right) \\
& =-\int_{\partial Y} \operatorname{tr}\left(d_{A_{0}} \xi \wedge d_{A_{0}} \xi^{\prime}\right) \\
& =-\int_{\partial Y} \operatorname{tr}\left(\xi\left[F_{A_{0}}, \xi^{\prime}\right]\right)=0 .
\end{aligned}
$$

The last integral equals zero because $A_{0}$ is flat on $\partial Y$. Our nondegeneracy assumptions guarantee that $\lambda^{\prime}(0) \neq 0$.

Finally, we relate the sign of the spectral flow $\frac{\lambda^{\prime}(0)}{\mid \lambda^{\prime}(0)}$ to the orientation of the irreducible arc limiting to the bifurcation point. A slice to the gauge group action through $A_{0}$ is

$$
X_{A_{0}}=\left\{A_{0}+a \mid d_{A_{0}}^{*} a=0, a \in \Omega_{\nu}^{1}(Y ; s u(2))\right\} .
$$


Let $\Pi_{\text {ker } d_{A_{0}}^{*}}$ denote the orthogonal projection from $\Omega^{1}(Y ; s u(2))$ onto ker $d_{A_{0}}^{*}$. By standard arguments involving the Kuranishi map, the connections in $X_{A_{0}}$ near $A_{0}$ for which $\Pi_{\text {ker } d_{A_{0}}^{*}} \zeta_{h}(A) \in \mathcal{H}_{A_{0}, h}^{1}(Y, \partial Y ; s u(2))$ make up a smooth 3-dimensional submanifold, parametrized by

$$
A(t, x, y)=A_{0}+t \vec{a}+x \vec{b}+y J \vec{c}+\phi(t, x, y),
$$

where $\phi: \mathbf{R}^{3} \rightarrow * d_{A_{0}, h} \Omega_{\tau}^{1}(Y ; s u(2))$ satisfies $\phi(0,0,0)=0$. See [H1], for example, for details.

To first order in $t, x$, and $y$, in terms of the bases $\{\vec{a}, \vec{b}, J \vec{b}\}$ and $\{\vec{u}, \vec{c}, J \vec{c}\}$ for $\mathcal{H}_{A_{0}, h}^{1}(Y ; s u(2))$ and $\mathcal{H}_{A_{0}}^{0}(Y ; s u(2)) \oplus \mathcal{H}_{A_{0}, h}^{1}(Y, \partial Y ; s u(2))$, the restricted operator has the form

$$
\Pi_{0} \circ K_{h}(A(t, x, y))=\left[\begin{array}{ccc}
0 & 2 y & -2 x \\
\lambda^{\prime}(0) x & \lambda^{\prime}(0) t & 0 \\
\lambda^{\prime}(0) y & 0 & \lambda^{\prime}(0) t
\end{array}\right] .
$$

The lower right hand $2 \times 2$ block in the above matrix is equal to $H_{t}$. The second and third entries in the first column must have the values given because of the fact that the bottom two rows form the derivative matrix for a map from $\mathcal{H}_{A_{0}, h}^{1}(Y ; s u(2))$ to $\mathcal{H}_{A_{0}, h}^{1}(Y, \partial Y ; s u(2))$. The top row entries are clear from the fact that $\operatorname{Stab}\left(A_{0}\right)$ acts with weight two on the complex coefficients.

For the purposes of keeping track of orientations this first order approximation is sufficient. By Theorem 12 of [H2], the irreducible perturbed flat connections in the slice near $A_{0}$, together with $A_{0}$ (which is reducible), form a 2-dimensional submanifold through $A_{0}$, invariant under the $\operatorname{Stab}\left(A_{0}\right)$ action, described to first order by $A(0, x, y)$. What remains for us to do is to identify the induced orientation on the radial direction in this tangent space from the orientation on the index of $K_{h}$.

We will say that two bases for a vector space are equivalent if they induce the same orientation on the vector space, and we denote this equivalence by $\sim$. Recall that by assumption, a correctly oriented basis for ker $K_{h}\left(A_{0}\right) \oplus$ $\operatorname{coker} K_{h}\left(A_{0}\right)$ is

$$
\{\vec{a}, \vec{b}, J \vec{b}, \vec{u}, \vec{c}, J \vec{c}\} .
$$

The corresponding basis for $\operatorname{Ind} K_{h}(A(0, x, 0))$ is $\{\epsilon \vec{b}, J \vec{c}\}$ where $\epsilon \in\{ \pm 1\}$ makes

$$
\begin{aligned}
& \left\{\epsilon \vec{b}, \vec{a}, J \vec{b}, J \vec{c}, \Pi_{0} \circ K_{h}(A(0, x, 0)) \vec{a}, \Pi_{0} \circ K_{h}(A(0, x, 0)) J \vec{b}\right\} \\
\sim & \left\{\epsilon \vec{b}, \vec{a}, J \vec{b}, J \vec{c}, \lambda^{\prime}(0) x \vec{c}, 2 x \vec{u}\right\} \\
\sim & \left\{\vec{a}, \lambda^{\prime}(0) \epsilon \vec{b}, J \vec{b}, \vec{u}, \vec{c}, J \vec{c}\right\}
\end{aligned}
$$


equivalent to

$$
\{\vec{a}, \vec{b}, J \vec{b}, \vec{u}, \vec{c}, J \vec{c}\} .
$$

In other words, $\epsilon=\frac{\lambda^{\prime}(0)}{\lambda^{\prime}(0)}$, so the sign coincides with the spectral flow direction of $H_{t}$. This is summarized in the following proposition.

Proposition 4.2. The orientation on the irreducible arc limiting to a bifurcation point points away from or towards the bifurcation point according to whether the spectral flow of $H_{t}$ is positive or negative.

Remark: By Corollary 3 of [H2], the direction in $\mathcal{M}_{T^{2}}$ in which $r\left(\mathcal{M}_{h}^{*}\right)$ leaves the image of the bifurcation point is also determined by this sign. Thus the orientation on the non-closed components of $\tilde{\mathcal{M}}_{h}^{*}$ is determined by their image in $\tilde{\mathcal{M}}_{T^{2}}$.

\section{An Intersection Formula.}

Choose a 0 -framed longitude $\lambda$ for the knot, i.e., an embedded circle in $\partial Y$ which is null homologous in $Y$. Also, choose a meridian curve $\mu$, the boundary of a normal disk in $\nu(\kappa)$. We require that $\lambda$ and $\mu$ are oriented so that their intersection is $\mu \cdot \lambda=1$, using the boundary orientation on $T^{2}$. Note that this orients the index bundles from Section 2 on both $Y$ and $S^{1} \times D^{2}$, since $\lambda$ and $\mu$ generate $H^{1}\left(S^{1} \times D^{2} ; \mathbf{R}\right)$ and $H^{1}(Y ; \mathbf{R})$, respectively.

For each $0 \leq \alpha<\pi$, define

$$
\left.\tilde{S}_{\alpha}=\left\{[A] \in \tilde{\mathcal{M}}_{T^{2}} \mid \operatorname{hol}_{\mu}(A)\right)=\exp (\mathbf{i} \alpha)\right\} .
$$

Orient this circle so that $\tilde{r}\left(\tilde{\mathcal{M}}^{U(1)}\right) \cdot \tilde{S}_{\alpha}=1$. For $\alpha=0, \tilde{S}_{0}=r\left(\tilde{\mathcal{M}}_{S^{1} \times D^{2}}\right)$, and this orientation on $\tilde{S}_{0}$ is corresponds to the one on $\tilde{\mathcal{M}}_{S^{1} \times D^{2}}$ coming from the choice of first homology generator $\lambda$.

Assume that the map

$$
\phi: \tilde{\mathcal{M}}_{h}^{U(1)} \rightarrow U(1)
$$

given by $\phi([A])=\operatorname{hol}_{\mu}(A)$ is a diffeomorphism. (Large perturbations can violate this requirement, but small ones cannot.) For each bifurcation point $[A] \in \tilde{\mathcal{M}}_{h}^{U(1)}$, let $\sigma([A])= \pm 1$ depending on whether the eigenvalue $\lambda(t)$ of $H_{t}$ has positive or negative derivative when it crosses zero.

Define $\mathcal{S}:(0, \pi) \rightarrow \mathbf{Z}$ to be $\mathcal{S}(\alpha)=\sum \sigma([A])$ where the sum is taken over all bifurcation points in $\phi^{-1}(0, \alpha)$.

For each $0 \leq \alpha<\pi$, let $A^{\alpha}$ denote a flat abelian connection on $Y$ with meridinal holonomy equal to $\exp (\mathrm{i} \alpha) \in S U(2)$. Such a connection is unique up to gauge equivalence. 
Lemma 5.1. Let $0<\alpha<\pi$ be a number such that $\mathcal{H}_{A^{\alpha}}^{1}(Y, \partial Y ; s u(2))=0$. Let $h$ be a perturbation such that the following conditions hold.

(1) $\mathcal{M}_{h}$ is nondegenerate.

(2) $\tilde{S}_{\alpha}$ meets $\tilde{r}\left(\tilde{\mathcal{M}}_{h}^{*}\right)$ transversely.

(3) $\tilde{S}_{\alpha}$ misses the bifurcation points of $\tilde{r}\left(\tilde{\mathcal{M}}_{h}^{U(1)}\right)$.

(4) $r$ maps $\mathcal{M}_{h}^{U(1)}$ onto $r\left(\mathcal{M}_{h_{0}}^{U(1)}\right)$.

(5) There is a path $h_{t}$ of perturbations from 0 to $h$ such that $\mathcal{M}_{h_{t}}$ satisfies (1), (3) and (4) for all $0<t \leq 1$.

Then

$$
r\left(\tilde{\mathcal{M}}_{h}^{*}\right) \cdot \tilde{S}_{\alpha}-r\left(\tilde{\mathcal{M}}_{h}^{*}\right) \cdot \tilde{S}_{0}=\mathcal{S}(\alpha)
$$

Remark: We will show in Section 3 that it is always possible to find a small perturbation satisfying $(1)-(5)$.

Proof. of Lemma 5.1 This reduces to the following elementary topological fact. Let $\Sigma \subset \tilde{\mathcal{M}}_{T^{2}}$ denote the smaller annulus between $\tilde{S}_{0}$ and $\tilde{S}_{\alpha}$, i.e., $\Sigma=\bigcup_{0 \leq \beta \leq \alpha} \tilde{S}_{\beta}$. The induced orientation makes $\partial \Sigma=\tilde{S}_{\alpha}-\tilde{S}_{0}$.

For any immersion $f: C \rightarrow \tilde{\mathcal{M}}_{T^{2}}$ of a compact, oriented, 1-dimensional submanifold $C$ with $f(\partial C) \cap \partial \Sigma=\emptyset$ and $f(C)$ transverse to $\partial \Sigma, f(C)$. $\partial \Sigma$ equals the number of endpoints (counted with orientation) of $C$ whose images lie in $\Sigma$.

\section{6. $\mathcal{S}(\alpha)$ and the Equivariant Knot Signature.}

We next relate $\mathcal{S}(\alpha)$ to the spectral flow of the twisted signature operator on the 0-surgery on $\kappa$. We then relate this spectral flow to the signature of the matrix $B_{k}\left(e^{i 2 \alpha}\right)$. We begin by recalling the definition of spectral flow for a family of self-adjoint Fredholm operators.

Let $K_{t}, 0 \leq t \leq 1$, be a continuous 1-parameter family of self-adjoint Fredholm operators on a separable Hilbert space. The eigenvalues of $K_{t}$ are real and vary continuously in $t$. We consider their graphs in $[0,1] \times \mathbf{R}$. The nonzero eigenvalues of $K_{0}$ and $K_{1}$ are bounded away from zero. Let 
$\delta>0$ be smaller than the absolute value of the nonzero eigenvalue closest to zero. A small homotopy of the family of operators, fixing the endpoints, will insure that the eigenvalues cross this line transversely. Then we define the spectral flow $S F\left(K_{0}, K_{1}\right)$ to be the number of eigenvalues, counted with multiplicities, which cross the line segment connecting $(0,-\delta)$ to $(1, \delta)$; an eigenvalue counts with a plus or minus sign depending on whether it crosses from below to above or from above to below. Equivalently, the spectral flow from $K_{0}$ to $K_{1}$ is the number of eigenvalues that change from non-negative to non-positive. Note that with this convention, $S F\left(K_{0}, K_{1}\right)+S F\left(K_{1}, K_{2}\right)=$ $S F\left(K_{0}, K_{2}\right)-\operatorname{dim} \operatorname{ker}\left(K_{1}\right)$.

Let $\bar{Y}=Y \cup_{\partial Y} S^{1} \times D^{2}$ be the closed manifold obtained by 0-surgery on $\kappa$. We will denote connections on $\bar{Y}$ by $\bar{A}$. In this section we assume that $h$ is a perturbation such that $\mathcal{M}_{h}(Y)$ satisfies (1) - (5). We are considering $h$ as a perturbation for both $Y$ and $\bar{Y}$. Condition (4) insures that the abelian portion of $\mathcal{M}_{h}(\bar{Y})$ is a 1-dimensional arc parametrized by $\operatorname{tr}\left(\operatorname{hol}_{\mu}(\bar{A})\right.$ ). (This is not the case for generic perturbations on $\bar{Y}$, but it can be arranged by choosing the perturbations in $Y$ as prescribed above.)

Let $K_{\bar{A}, h}: \Omega^{0}(\bar{Y} ; s u(2)) \oplus \Omega^{1}(\bar{Y} ; s u(2)) \rightarrow \Omega^{0}(\bar{Y} ; s u(2)) \oplus \Omega^{1}(\bar{Y} ; s u(2))$ be the twisted signature operator for $\bar{A}$ on $\bar{Y}$, given by

$$
K_{\bar{A}, h}(\sigma, \tau)=\left(d_{\bar{A}}^{*} \tau, d_{\bar{A}} \sigma+* d_{\bar{A}, h} \tau\right) .
$$

Let $A_{t}$ be a path of abelian connections on $Y$ such that $\left[A_{t}\right]$ passes through a bifurcation point when $t=0$, as in Section 4 . Let $\bar{A}_{t}$ be a family of abelian connections on $\bar{Y}$ such that $\left.\bar{A}_{t}\right|_{Y}=A_{t}$ and $\left.\bar{A}_{t}\right|_{S^{1} \times D^{2}}$ is flat. Let $\bar{a}$ denote the tangent vector $\left.\frac{d}{d t} \bar{A}_{t}\right|_{t=0}$. The next proposition relates the spectral flow of the finite dimensional self-adjoint operator $H_{t}$ defined in Section 1 to the spectral flow of the operator $K_{\bar{A}_{t}, h}$.

Proposition 6.1. As t passes through 0 , exactly one eigenvalue of $K_{\bar{A}_{t}, h}$, having multiplicity two, changes sign, and it changes in the same direction as $\lambda(t)$.

Proof. The Mayer Vietoris sequence implies that $\bar{Y}$ has a jump in (twisted) first cohomology at exactly those abelian connections which restrict to be bifurcation points on $Y$. The only problem is to relate the sign of the spectral flows at these points.

The Mayer Vietoris sequence along with our assumption about $\mathcal{M}_{h}^{U(1)}$ together imply that the restriction map from $\mathcal{H}_{\bar{A}, h}^{1}(\bar{Y} ; s u(2))$ to $\mathcal{H}_{A, h}^{1}(Y ; s u(2))$ is an isomorphism. 
Let $\bar{\lambda}(t)$ be the eigenvalue of $K_{\bar{A}_{t}, h}$ which passes through 0 when $t=0$, and let $\bar{\phi}(t)$ be a corresponding eigenvector. Then $\bar{\phi}(0) \in \mathcal{H} \frac{1}{A, h}(\bar{Y} ; \mathbf{C})$, and in fact $\bar{\phi}(t)$ can be chosen so that $\left.\bar{\phi}(0)\right|_{Y}=\vec{b}$.

$$
\left\langle K_{\bar{A}_{t}, h} \bar{\phi}(t), \bar{\phi}(t)\right\rangle=\bar{\lambda}(t)\|\bar{\phi}(t)\|_{L^{2}(\bar{Y})}^{2} .
$$

Differentiating in $t$ and evaluating when $t=0$ gives

$$
\bar{\lambda}(t)^{\prime}(0)\|\bar{\phi}(0)\|_{L^{2}(\bar{Y})}^{2}=\langle *[\bar{a} \wedge \bar{\phi}(0)], \bar{\phi}(0)\rangle=-\int_{\bar{Y}} \operatorname{tr}([\bar{a} \wedge \bar{\phi}(0)] \wedge \bar{\phi}(0)) .
$$

(Note: this pairing only depends on the cohomology classes of $\bar{\phi}(0)$ and $\bar{a}$.)

Breaking up the integral we obtain

$$
\begin{aligned}
-\int_{\bar{Y}} \operatorname{tr}([\bar{a} \wedge \bar{\phi}(0)] \wedge \bar{\phi}(0))= & -\int_{Y} \operatorname{tr}([\vec{a} \wedge \bar{\phi}(0)] \wedge \bar{\phi}(0)) \\
& -\int_{S^{1} \times D^{2}} \operatorname{tr}([\bar{a} \wedge \bar{\phi}(0)] \wedge \bar{\phi}(0)) \\
= & -\int_{Y} \operatorname{tr}([\vec{a} \wedge \vec{b}] \wedge \vec{b}) .
\end{aligned}
$$

The integral over the solid torus vanishes because there $\bar{\phi}_{0}$ is exact and $\bar{a}$ is closed. The integral over $Y$ has the same sign as $\lambda^{\prime}(0)$.

Corollary 6.2. $2 \mathcal{S}(\alpha)-4=S F\left(K_{\bar{A}^{0}, h}, K_{\bar{A}^{\alpha}, h}\right)(\bar{Y})$.

Proof. The image of the set of bifurcation points in $\mathcal{M}_{h_{t}}, 0<t \leq$ 1 , in $\mathcal{M}_{T^{2}}$ is bounded away from the trivial orbit. It follows that $\operatorname{dim} \operatorname{ker} K_{\bar{A}^{0}, h_{t}}(\bar{Y})$ is constantly equal to 6 , and that for $\beta>0$ very small $\operatorname{dim} \operatorname{ker} K_{\bar{A}^{\beta}, h_{t}}$ is constantly equal to 2 for $0 \leq t \leq 1$. Therefore $S F\left(K_{\bar{A}^{0}, h}, K_{\bar{A}^{\beta}, h}\right)(\bar{Y})=S F\left(K_{\bar{A}^{0}}, K_{\bar{A}^{\beta}}\right)(\bar{Y})$ which is easily calculated to be -4 by explicitly computing $\left.K_{\bar{A}^{\beta}}\right|_{\operatorname{ker} K_{\bar{A}^{0}}}$. The proposition implies that $2 \mathcal{S}(\alpha)=S F\left(K_{\bar{A}^{\beta}, h}, K_{\bar{A}^{\alpha}, h}\right)(\bar{Y})+2$, which equals $S F\left(K_{\bar{A}^{0}}, K_{\bar{A}^{\alpha}}\right)(\bar{Y})+4$ by the above argument.

We now relate the spectral flow of the twisted signature operator on $\bar{Y}$ to the signature of the matrix $B_{\kappa}\left(e^{i 2 \alpha}\right)=\left(1-e^{i 2 \alpha}\right) V+\left(1-e^{-i 2 \alpha}\right) V^{T}$, where $V$ is the Seifert pairing of a Seifert surface for $\kappa$. The following modification of the argument in [KKR], Section 4, was suggested to the author by Paul Kirk. 
Proposition 6.3. $S F\left(K_{A^{0}}, K_{A^{\alpha}}\right)(\bar{Y})=-\operatorname{Sign} B_{\kappa}\left(e^{i 2 \alpha}\right)-4$.

Proof. We will use a formula relating the spectral flow of $K_{A}$ on $\bar{Y}$ to the Chern-Simons function and rho invariants of the flat connections at the ends of the path. A derivation of this formula may be found in [KKR].

Let $A^{\alpha}$ and $A^{\beta}$ be any flat connections on a 3-manifold $Z$ with holonomy representations $\alpha, \beta: \pi_{1} Z \rightarrow S U(2)$. The formula is the following:

$$
\begin{aligned}
S F( & \left.K_{A^{\alpha}}, K_{A^{\beta}}\right)(Z) \\
= & 8\left(\operatorname{cs}\left(A^{\beta}\right)(Z)-c s\left(A^{\alpha}\right)(Z)\right) \\
& +\frac{1}{2}\left(\rho_{a d \beta}(Z)-\rho_{a d \alpha}(Z)\right) \\
& -\frac{1}{2}\left(\operatorname{dim} \operatorname{ker} K_{A^{\alpha}}+\operatorname{dim} \operatorname{ker} K_{A^{\beta}}\right) .
\end{aligned}
$$

Suppose that $M$ is an oriented 4-manifold with oriented boundary $Z$. Suppose furthermore that $\tilde{\alpha}: \pi_{1} M \rightarrow S U(2)$ is an extension of the complexified adjoint representations $a d \alpha$. Then the rho invariant of $a d \alpha$ may be calculated with the formula

$$
\rho_{a d \alpha}(Z)=3 \operatorname{Sign} M-\operatorname{Sign}_{\tilde{\alpha}} M,
$$

where the latter term is the equivariant signature of $M$.

We begin by describing a 4 -manifold to which we will apply the above spectral flow formula. Let $U$ be the union of $X \times[0,1]$ with a 2-handle attached to $X \times\{1\}$ along $N(\kappa)$ with the 0 -framing. Orient $U$ so that $\partial U=\bar{Y}-X$.

Let $F$ be a Seifert surface for $\kappa \times\{1\}$, the interior of which is pushed into $X \times[0,1]$. Let $\bar{F}$ denote the closed surface obtained by capping $F$ off with the core of the 2-handle. $\bar{F}$ has trivial normal bundle in $U$, so we may remove $N(\bar{F})$ and replace it by $S^{1} \times H$ where $H$ is a handlebody of genus equal to that of $\bar{F}$. Call the resulting manifold $M$.

Proposition 6.4. $\quad$ 1. $H_{1}(M)=\mathrm{Z}$.

2. The map $H_{1}(Y) \rightarrow H_{1}(M)$ induced by the inclusion map is an isomorphism.

3. $\operatorname{Sign}(M)=0$. 
This proposition is just a homology calculation, so it suffices to show it for the case of $\kappa$ equal to the unknot in $S^{3}$, with $F$ equal to a disk. The claims are easily checked in this case.

The important property of $M$ is that $\partial M=\bar{Y}-X$ and any abelian representation $\alpha: \pi_{1} \bar{Y} \rightarrow S U(2)$ extends uniquely to an abelian representation $\tilde{\alpha}$ of $\pi_{1} M$ which is trivial on $\pi_{1} X$. Thus $\rho_{a d \alpha}(\bar{Y})-\rho_{\text {trivial }}(X)=$ $3 \operatorname{Sign} M-\operatorname{Sign}_{\tilde{\alpha}} M$. Since the Chern-Simons function is a flat cobordism invariant, $c s\left(A^{\alpha}\right)(\bar{Y})=c s\left(A^{0}\right)(X)=0$, and the Chern-Simons function terms in the spectral flow formula contribute zero.

The formula then implies

$$
S F\left(K_{A^{0}}, K_{A^{\alpha}}\right)(\bar{Y})=-\frac{1}{2} \operatorname{Sign}_{\tilde{\alpha}} M-4 .
$$

Proposition 6.3 is now a consequence of the following fact.

Proposition 6.5. $\operatorname{Sign}_{\tilde{\alpha}} M=2 \operatorname{Sign} B_{\kappa}\left(e^{i 2 \alpha}\right)$.

Proof. of Proposition 6.5 Since $S^{1} \times H$ has a deformation retract $S^{1} \times\left(\vee_{i} S^{1}\right)$ in its boundary, $\operatorname{Sign} S^{1} \times H$ and $\operatorname{Sign}_{\tilde{\alpha}} S^{1} \times H$ are both zero. By Novikov additivity $\operatorname{Sign}_{\tilde{\alpha}} M=\operatorname{Sign}_{\tilde{\alpha}} U \backslash N(F)$.

Let $N=U \backslash N(F)$, and let $\tilde{N}$ be the universal abelian cover of $N$. Let $B: H_{2}(\tilde{N} ; \mathbf{Z}) \times H_{2}(\tilde{N} ; \mathbf{Z}) \rightarrow \mathbf{Z}\left[t, t^{-1}\right]$ denote the equivariant intersection form.

Theorem 6.6. (Theorem 4.2 of $[K K R]) H_{2}(\tilde{N} ; \mathbf{Z})=\left(\mathbf{Z}\left[t, t^{-1}\right]\right)^{2 g} \oplus \mathbf{Z}$ where $\mathbf{Z}\left[t, t^{-1}\right]$ acts trivially on the $\mathbf{Z}$ summand. The matrix for the equivariant intersection form, $B$, on the free summand is given by

$$
(1-t) V+\left(1-t^{-1}\right) V^{T}
$$

where $V$ is the Seifert matrix for the Seifert surface $F, V_{i j}=\ell k\left(x_{i}, x_{j}^{+}\right)$. The $\mathbf{Z}$ summand is in the kernel of $B$.

The proof of this theorem is a standard argument which may be found in Chapter VII of $[\mathrm{K}]$ and in [KKR]. The idea is to construct $\tilde{N}$ as the union of the universal cover of the 2-handle minus its core and the universal cover of $X \times[0,1] \backslash F$. In both of the above references, the calculation is done for the case when $X=S^{3}$ (and the trivial $X$ end of the cobordism $U$ is capped off with a 4-ball). It works the same for the present situation, however. The 
important feature is that $(X \times[0,1]) \backslash N(F)$ cut open along the trace of a homotopy from $F$ to a Seifert surface in $X \times\{1\}$ has no 1-dimensional or 2-dimensional cohomology.

All that remains to prove Proposition 6.5 is to understand the adjoint action of $\exp (\mathbf{i} \alpha)$ on the complexified Lie algebra $s u(2) \otimes \mathbf{C}$. The representation of $H_{1}(M)=\mathbf{Z}$ reduces to a direct sum of $3 U(1)$ representations, taking the generator to $e^{i 2 \alpha}, e^{-i 2 \alpha}$, and 1 . Thus

$$
\operatorname{Sign}_{\tilde{\alpha}} N=\operatorname{Sign} B_{\kappa}\left(e^{i 2 \alpha}\right)+\operatorname{Sign} B_{\kappa}\left(e^{-i 2 \alpha}\right)+\operatorname{Sign} B_{\kappa}(1)=+2 \operatorname{Sign} B_{\kappa}\left(e^{i 2 \alpha}\right) .
$$

\section{Casson's Invariant.}

In this section we relate the intersection number $\tilde{r}\left(\tilde{\mathcal{M}}_{h}^{*}\right) \cdot \tilde{S}_{0}$ to the Casson invariant. We will make use of the fact that Taubes showed $\lambda(X)=-\frac{1}{2} \sum_{[A] \in \mathcal{M}^{*(X)}}(-1)^{S F\left(K_{\ominus}, K_{A}\right)}$ in [T]; see also [KKR], Section 2, for clarification of the sign.

Theorem 7.1. $\tilde{r}\left(\tilde{\mathcal{M}}_{h}^{*}\right) \cdot \tilde{S}_{0}=-4 \lambda(X)$.

We first prove this equality up to an overall sign. That is, we show that the oriented intersection number $\tilde{r}\left(\tilde{\mathcal{M}}_{h}^{*}\right) \cdot \tilde{S}_{0}$ has the same absolute value as 4 times Casson's invariant for $X$.

Proposition 7.2. Let $[A]$ and $\left[A^{\prime}\right]$ be orbits in $\tilde{\mathcal{M}}_{h}^{*} \cap \tilde{r}^{-1}\left(\tilde{S}_{0}\right)$. Then the local intersection numbers of $\tilde{r}\left(\tilde{\mathcal{M}}_{h}^{*}(Y)\right) \cdot \tilde{S}_{0}$ at $[A]$ and $\left[A^{\prime}\right]$ agree if and only if $S F\left(K_{A, h}, K_{A^{\prime}, h}\right)$ equals zero modulo 2.

Remark: Both of the quantities are unchanged when $A^{\prime}$ is replaced by $g\left(A^{\prime}\right)$ for any gauge transformation. Thus it is sufficient to prove the proposition for one for arbitrary $A \in \zeta_{h}^{-1}(0)$ and for any representative $A^{\prime}$ of an arbitrary orbit in the intersection. In fact, it is enough to do so for pairs $[A],\left[A^{\prime}\right]$ in the same component of $\tilde{r}^{-1}\left(\tilde{S}_{0} \backslash\{\right.$ centrals $\left.\}\right)$.

Proposition 7.2 is analogous with Proposition 5.2 of [T], with the following difference. Taubes was concerned with intersecting the two irreducible strata of the flat moduli spaces for the solid handlebodies in a Heegard decomposition for $X$ in the irreducible stratum of the flat moduli space for the splitting surface. Because our connections are abelian on the torus $\partial Y$, 
and on the tubular neighborhood of the knot, the Fredholm operators which for Taubes were surjective have cokernel in our case. We have already dealt with this problem Section 2 and we now modify Taubes' argument to include the identifications of these two cokernels with $\mathcal{H}^{0}(\partial Y ; s u(2))$.

We begin by establishing some notation that will parallel that in $[\mathrm{T}]$. Recall that $X$ is a homology 3-sphere, in which $Y$ sits as complement of an open tubular neighborhood of a knot. Let $X_{1}=Y$ and let $X_{2}=S^{1} \times D^{2}$ be the closure of a slightly larger tubular neighborhood, so that $X_{0}=X_{1} \cap X_{2}$ is diffeomorphic to a product $T^{2} \times[0,1]$. We may assume that the perturbation curves do not intersect $X_{0}$.

We make the following definition

$$
\mathcal{A}^{\diamond}=\left\{A \in \mathcal{A}(X)|A|_{X_{2}} \in \Omega^{1}\left(X_{2} ; u(1)\right),\left.A\right|_{X_{2}} \text { is not central }\right\} .
$$

For each $A \in \mathcal{A}^{\diamond}$, let $\mathcal{T}_{\alpha}(A)=\operatorname{ker} d_{A}^{*} \cap \Omega_{\nu}^{1}\left(X_{\alpha}\right)$. These fit together to form bundles $\mathcal{T}_{\alpha}$ over $\mathcal{A}^{\diamond}$. For $\alpha=0,2$, this requires a straightforward modification of the usual argument, taking advantage of the fact that ker $d_{A} \cap \Omega^{0}$ is always the span of the constant 0 -form $\mathbf{i}$.

The bundles fit into an exact, acyclic Fredholm complex

$$
0 \rightarrow \mathcal{T} \stackrel{\Phi}{\rightarrow} \mathcal{T}_{1} \oplus \mathcal{T}_{2} \stackrel{\Psi}{\rightarrow} \mathcal{T}_{0} \rightarrow 0 .
$$

The map $\Phi$ is defined by

$$
\Phi(a)=\left(\left.a\right|_{X_{1}}-d_{A} b_{1},\left.a\right|_{X_{2}}-d_{A} b_{2}\right)
$$

where $b_{1,2}$ is the unique 0 -form satisfying

$$
d_{A}^{*} d_{A} b_{1,2}=0,\left.* d_{A} b_{1,2}\right|_{\partial X_{1,2}}=\left.* a\right|_{\partial X_{1,2}}
$$

and $b_{2} \perp \mathbf{i}$.

The map $\Psi$ is defined by

$$
\Psi\left(a_{1}, a_{2}\right)=\left.a_{1}\right|_{X_{0}}-\left.a_{2}\right|_{X_{0}}-d_{A} b_{0}
$$

where $b_{0}$ is the unique 0 -form satisfying

$$
d_{A}^{*} d_{A} b_{0}=0,\left.* d_{A} b_{0}\right|_{\partial X_{0}}=\left.*\left(a_{1}-a_{2}\right)\right|_{\partial X_{0}}
$$

and $b_{0} \perp \mathbf{i}$.

Next, define $\mathcal{L}_{\alpha}=\operatorname{ker} d_{A}^{*} \cap \Omega^{1}\left(X_{\alpha} ; s u(2)\right)$ for $\alpha=\emptyset, 0,1,2$. Again we have an exact, acyclic, Fredholm complex

$$
0 \rightarrow \mathcal{L} \stackrel{\bar{\Phi}}{\rightarrow} \mathcal{L}_{1} \oplus \mathcal{L}_{2} \stackrel{\bar{\Psi}}{\rightarrow} \mathcal{L}_{0} \rightarrow 0,
$$


where the maps are $\bar{\Phi}(a)=\left(\left.a\right|_{X_{1}},\left.a\right|_{X_{2}}\right)$ and $\bar{\Psi}\left(a_{1}, a_{2}\right)=\left.a_{1}\right|_{X_{0}}-\left.a_{2}\right|_{X_{0}}$.

Proof of Proposition 7.2: Let $A, A^{\prime} \in \mathcal{A}^{\diamond}$ be two perturbed flat irreducible connections on $X$. We will use the same notation for their restrictions to the pieces $X_{\alpha}$. Without loss of generality, we can assume that $A$ and $A^{\prime}$ are connected by a path $A_{t}, t \in[0,1]$ for which $\left.A_{t}\right|_{X_{2}}$ is flat and which is mapped 1-1 onto the arc in $\tilde{S}_{0}$ connecting $\left[\left.A\right|_{\partial X_{1}}\right]$ to $\left[\left.A^{\prime}\right|_{\partial X_{1}}\right]$ which does not include any central orbits.

To conserve notation, let $\mathcal{T}_{\alpha}(t)$ and $\mathcal{L}_{\alpha}(t)$ denote the fibers of these bundles over $A_{t}$. We will let $B_{\alpha}(t)$ denote $\left.* d_{A_{t}, h}\right|_{\mathcal{T}_{\alpha}(t)}$ composed with orthogonal projection onto $\mathcal{L}_{\alpha}(t)$. We begin by considering the diagram of maps

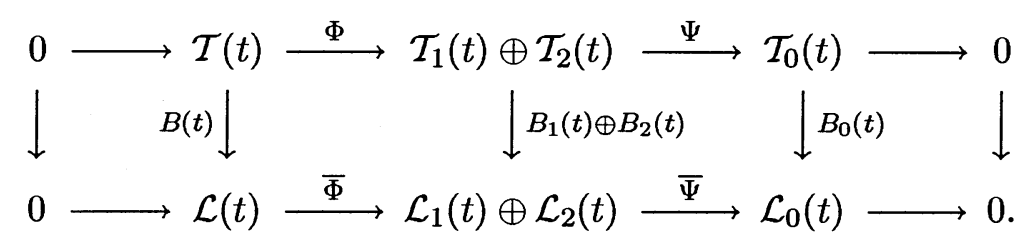

Next we adjust the complex to compensate for the extra cokernel at the perturbed flat connections. $B_{2}(t)$ is surjective for all $t$. When $t=0,1$, $B_{1}$ has cokernel equal to $\mathcal{H}_{A_{t}, h}^{1}\left(X_{1}, \partial X_{1} ; s u(2)\right)$. To eliminate this, we let $B_{1}^{\prime}(t): \mathcal{T}_{1}(t) \oplus \mathbf{R} \rightarrow \mathcal{L}_{1}(t)$ be the map $(a, s) \mapsto\left(B_{1}(a), s \Pi_{\mathcal{L}_{1}} d_{A_{t}} \mathbf{i}\right)$. Then, given our nondegeneracy assumptions on $h, B_{1}^{\prime} \oplus B_{2}$ is surjective for $t=0,1$. Similarly, for $t=0,1, B$ is an isomorphism but $B_{0}$ has cokernel equal to $\mathcal{H}_{A_{t}, h}^{1}\left(X_{0}, \partial X_{0} ; s u(2)\right) \cong \mathcal{H}_{A_{t}}^{2}\left(\partial X_{1} ; s u(2)\right)$. Let $B_{0}^{\prime}: \mathcal{T}_{0} \oplus \mathbf{R} \rightarrow \mathcal{L}_{0}$ be the map $(a, s) \mapsto\left(B_{0}(a), s \mathbf{i} d u\right)$ where $u$ is the $[0,1]$ variable.

Proposition 7.3. There exists a pair of nonzero numbers $\lambda(0)$ and $\lambda(1)$ of the same sign such that for $t=0,1$ the following diagram commutes:

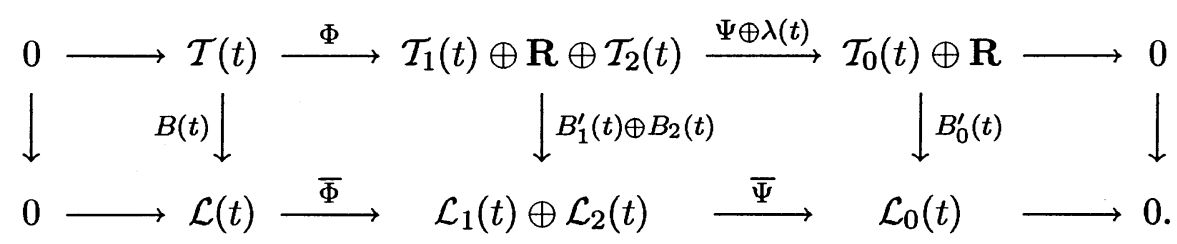

Proof. We will prove this by showing that for each $t \in[0,1], \bar{\Psi}\left(\Pi_{\mathcal{L}_{1}}\left(d_{A} \mathbf{i}\right)\right)$ is a nonzero multiple of $\mathbf{i} d u$. Let $A \in \mathcal{A}^{\diamond}(X)$ be a connection with $\left.A\right|_{X_{2}}$ flat. 
Let $b \in \Omega^{0}\left(X_{1} ; s u(2)\right)$ be the unique solution to the equations

$$
d_{A}^{*} d_{A} b=0,\left.b\right|_{\partial X_{1}}=0 .
$$

Then $\Pi_{\mathcal{L}_{1}} d_{A} \mathbf{i}=d_{A}(\mathbf{i}-b)$.

Suppose that $\left.\Pi_{\mathcal{L}_{1}} d_{A} \mathbf{i}\right|_{X_{0}}=0$. Then $\left.b\right|_{X_{0}}$ is a constant multiple of $\mathbf{i}$. But then the boundary conditions on $b$ imply that $\left.b\right|_{X_{0}}=0$, which implies that $b=0$ on $X_{1}$. This contradicts the fact that $d_{A}^{*} d_{A} \mathbf{i} \neq 0$ on $X_{1}$. Therefore $\left.\Pi_{\mathcal{L}_{1}} d_{A} \mathbf{i}\right|_{X_{0}} \neq 0$.

The preceding observation rules out the possibility that $\left(\Pi_{\mathcal{L}_{1}} d_{A} \mathbf{i}, 0\right)=$ $\bar{\Phi}(a)$ for some $a \in \mathcal{L}$. By exactness, $\bar{\Psi}\left(\Pi_{\mathcal{L}_{1}} d_{A} \mathbf{i}, 0\right) \neq 0$. We conclude the proof of Proposition 7.3 by showing that $\left.\Pi_{\mathcal{L}_{1}} d_{A} \mathbf{i}\right|_{X_{0}} \perp * d_{A} \Omega^{1}\left(X_{0} ; s u(2)\right)$. Here we are making use of the Hodge decomposition theorem, justified by the fact that $A$ is flat on $X_{0}$, and the fact that $\mathcal{H}_{A}^{1}\left(X_{0} ; s u(2)\right)$ is generated by $\mathbf{i} d u$.

Let $z \in \Omega^{1}\left(X_{0} ; s u(2)\right)$.

$$
\begin{aligned}
\left\langle d_{A}(\mathbf{i}-b), * d_{A} z\right\rangle & =-\int_{X_{0}} \operatorname{tr}\left(d_{A}(\mathbf{i}-b) \wedge d_{A} z\right) \\
& =-\int_{\partial X_{0}} \operatorname{tr}\left((\mathbf{i}-b) \wedge d_{A} z\right) \\
& =-\int_{\partial X_{0}} \operatorname{tr}\left(\mathbf{i} d_{A} z\right)=\int_{X_{0}} \operatorname{tr}\left(d_{A} \mathbf{i} \wedge z\right)=0 .
\end{aligned}
$$

Now extend $\lambda:\{0,1\} \rightarrow \mathbf{R} \backslash\{0\}$ to a continuous function; for example, we can choose $\lambda(t)$ to be the number which satisfies $\bar{\Psi}\left(\Pi_{\mathcal{L}_{1}(t)} d_{A_{t}} \mathbf{i}, 0\right)=\lambda(t) \mathbf{i} d u$. We can now form the diagram in 7.3 for all $t \in[0,1]$. The rows are exact (since $\lambda(t)$ never vanishes) and the diagram commutes at the endpoints.

Choose a splitting $\bar{\Theta}(t): \mathcal{L}_{0}(t) \rightarrow \mathcal{L}_{1}(t) \oplus \mathcal{L}_{2}(t)$ with the property that $\bar{\Psi}(t) \circ \bar{\Theta}(t)=1$. Since the vertical maps in the diagram are surjective for $t=0,1$, one can easily construct splittings $\Theta(t): \mathcal{T}_{0}(t) \oplus \mathbf{R} \rightarrow \mathcal{T}_{1}(t) \oplus \mathbf{R} \oplus \mathcal{T}_{2}$ such that $\left(B_{1}^{\prime}(t) \oplus B_{2}(t)\right) \circ \Theta(t)=\bar{\Theta} \circ B_{0}^{\prime}(t)$ for $t=0,1$. Since the set of all splittings of this short exact sequence may be identified with the space of bounded maps from $\mathcal{T}_{0} \oplus \mathbf{R}$ to $\mathcal{T}$, which is connected, these may be connected by a path of splittings $\Theta(t): \mathcal{T}_{0}(t) \oplus \mathbf{R} \rightarrow \mathcal{T}_{1}(t) \oplus \mathbf{R} \oplus \mathcal{T}_{2}(t)$ satisfying $(\Psi \oplus \lambda(t)) \circ \Theta(t)=1$.

We shift our attention to the folded over diagram given below. 


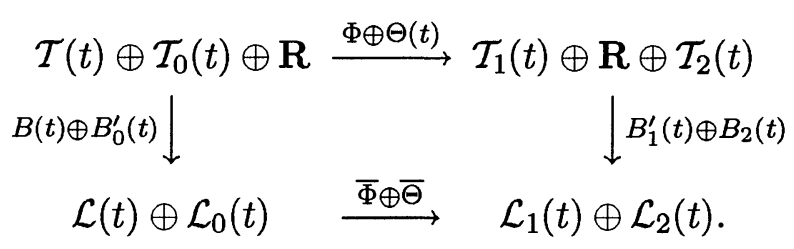

Its rows are isomorphisms. Furthermore, the diagram commutes at perturbed flat connections (i.e., for $t=0,1$ ). The columns are operators of index 2 , and when $t=0,1$ they are surjective. The operator $B_{0}^{\prime}(t)$ has is always surjective, with kernel equal to $\mathcal{H}_{A_{t}}^{1}\left(X_{0} ; s u(2)\right)$, which we may identify with $\mathbf{R}^{2}$. Let $\Pi_{1}: \mathcal{T}_{0}(t) \rightarrow \mathbf{R}^{2}$ denote the orthogonal projection onto this subspace.

We use this to make one final diagram from the previous one, as follows.

$$
\begin{array}{rll}
\mathcal{T}(t) \oplus \mathcal{T}_{0}(t) \oplus \mathbf{R} \stackrel{\Phi \oplus \Theta(t)}{\longrightarrow} \mathcal{T}_{1}(t) \oplus \mathbf{R} \oplus \mathcal{T}_{2}(t) \\
B(t) \oplus B_{0}^{\prime}(t) \oplus \Pi_{1} \downarrow & \downarrow B_{1}^{\prime}(t) \oplus B_{2}(t) \oplus\left(\Pi_{1} \circ \Psi(t)\right) \\
\mathcal{L}(t) \oplus \mathcal{L}_{0}(t) \oplus \mathbf{R}^{2} \stackrel{\bar{\Phi} \oplus \bar{\Theta} \oplus 1}{\longrightarrow} \mathcal{L}_{1}(t) \oplus \mathcal{L}_{2}(t) \oplus \mathbf{R}^{2} .
\end{array}
$$

The rows of this diagram are again isomorphisms, and the columns are when $t=0,1$. Furthermore, when $t=0,1$, it commutes.

Following [T], we let $\mathcal{F}_{n}$ denote the set of real, bounded, Fredholm operators on a separable Hilbert space which have index $n$, and let $\mathcal{F}_{n}^{1}$ denote the subset which has nonempty cokernel. $\mathcal{F}_{0}^{1}$ has codimension 1 in $\mathcal{F}^{1}$. Given two operators $F_{0}, F_{1} \in \mathcal{F}_{0}$, we define $\delta\left(F_{0}, F_{1}\right)$ to be the mod (2) cardinality of the set $\left\{F_{t}\right\} \cap \mathcal{F}_{0}^{1}$ for a generic path $F_{t}$ between them. This number is well defined and independent of the path $F_{t}$.

Applying this to the current situation, we get that the delta invariants of

$$
\begin{gathered}
\left(B_{1}^{\prime}(t) \oplus B_{2}(t) \oplus \Pi_{1}\right) \circ \Psi(t), \\
(\bar{\Phi}(t) \oplus \bar{\Theta}(t) \oplus 1) \circ\left(B(t) \oplus B_{0}^{\prime}(t) \oplus \Pi_{1} \circ \Psi(t)\right) \circ(\Phi(t) \oplus \Theta(t))^{-1}, \\
B(t) \oplus B_{0}^{\prime}(t) \oplus \Pi_{1} \text { and } B(t)
\end{gathered}
$$

are all equal. The last delta invariant is equal to $S F\left(K_{A_{0}}, K_{A_{1}}\right)(X)$ modulo 2 .

Finally, we relate the delta invariant of $B_{1}^{\prime}(t) \oplus B_{2}(t) \oplus \Pi \mathbf{R}^{2}$ to the relative intersections numbers at $\left[A_{0}\right]$ and $\left[A_{1}\right]$.

\section{Proposition 7.4.}


1. $B_{1}^{\prime}(t)$ is homotopic rel endpoints to a path $B_{1}^{\prime \prime}(t)$ which misses $\mathcal{F}_{1}^{1}$.

2. With $B_{1}^{\prime \prime}(t)$ chosen as above, $B_{1}^{\prime \prime}(t) \oplus B_{2}(t)$ is surjective for all $t$.

Proof. of Proposition 7.4: The first assertion follows immediately from Lemma 5.7 in $[\mathrm{T}]$. The second follows from the fact that $B_{2}(t)$ is surjective (recall that $\left.A_{t}\right|_{X_{2}}$ are flat $u(1)$ connections).

Now we conclude the proof of Proposition 7.2. Let $V_{t}=\operatorname{ker}\left(B_{1}^{\prime \prime}(t) \oplus\right.$ $\left.B_{2}(t)\right)$. These 2-dimensional subspaces fit together to form a vector bundle $V$ over $[0,1]$. The virtual vector bundle Index $B_{1}^{\prime}(t)$ is oriented in such a way that the orientation on $\operatorname{ker} B_{1}^{\prime}(t)$ agrees with that on $T_{\left[A_{t}\right]} \tilde{\mathcal{M}}_{h}$ for $t=$ 0,1 (compare with Section 2). Therefore $\operatorname{ker} B_{1}^{\prime \prime}(t)$, a 1-dimensional vector bundle over $[0,1]$, has the same property.

Let $\phi(t): V \rightarrow \mathbf{R}^{2}$ be the map $\phi(t)=\left.\Pi_{1} \circ \Psi(t)\right|_{V_{t}}$. Perturb $\phi(t)$ relative to the endpoints to get a $\phi^{\prime}(t): V \rightarrow \mathbf{R}^{2}$ with the section $\operatorname{det} \phi^{\prime}(t)$ transverse to the zero section of $\Lambda^{2}\left(\mathbf{R}^{2}\right) \otimes \Lambda^{2}\left(V^{*}\right)$. Then the relative orientations of $T_{\left[A_{t}\right]} \tilde{\mathcal{M}}_{h}^{*}\left(X_{1}\right) \oplus \tilde{S}_{0}$ with $\mathbf{R}^{2}$ at $t=0,1$ differ by the cardinality of $\operatorname{det}\left(\phi^{\prime}\right)^{-1}(0)$ modulo 2.

Proposition 7.2 proves Theorem 7.1 up to an overall sign. The remainder of this section is devoted to determining that sign.

Lemma 7.5. If $A$ is a perturbed flat connection on $X$ with $\mathcal{H}_{A, h}^{1}(X ; s u(2))$ trivial, and if $\Theta$ denotes the trivial connection, then $\tilde{r}\left(\tilde{\mathcal{M}}^{*}(Y)\right)$ intersects positively with $\tilde{r}\left(\tilde{\mathcal{M}}\left(S^{1} \times D^{2}\right)\right)$ if and only if the spectral flow from $K_{\Theta}$ to $K_{A}$ is even.

Proof. In the case where there are non-closed components in $\mathcal{M}_{h}^{*}$, we can perturb to deform $r\left(\mathcal{M}\left(S^{1} \times D^{2}\right)\right)$ until it intersects the image of an nonclosed arc such that $r\left(\mathcal{M}^{*}(Y)\right), r\left(\mathcal{M}^{U(1)}(Y)\right)$ and $r\left(\mathcal{M}\left(S^{1} \times D^{2}\right)\right)$ form a small triangle in $\mathcal{M}_{T^{2}}$. Then a direct spectral flow calculation (see [H3]) gives the allows the determination of the spectral flow from the trivial connection to the irreducible perturbed flat connection which is a corner of the triangle, which proves the theorem. If there is no such arc, we can produce one as follows.

On pp. 410-411 of [H1], we described a particular perturbation which gave rise to an irreducible arc in the perturbed flat moduli space of the solid torus. Performing the same perturbation using a pair of curves which are 
parallel to $\mu$ in $Y$ and have framing 1 does the same thing to the moduli space of $Y$ (adds a new non-closed component to $\mathcal{M}^{*}$ ). It also, however, creates singularities along each part of $\mathcal{M}_{h}^{*}$ which maps under $r$ into a neighborhood of $r\left(\mathcal{M}\left(S^{1} \times D^{2}\right)\right)$. By doing the construction from [H1] very close to the trivial orbit, however, and then by perturbing slightly on the Dehn filling, we can arrange that $r\left(\mathcal{M}\left(S^{1} \times D^{2}\right)\right)$ misses these new singularities. Furthermore, with a little care, we can arrange that the new intersections, except for two intersections with the new arc, correspond under a one-toone, orientation preserving map to the old intersections. Then, once again, the direct spectral flow calculation implies Lemma 7.5.

\section{Proof of Main Theorem.}

The proof of the main result of this paper now follows easily.

Theorem 8.1. For any $0<\alpha<\pi$ with $B_{\kappa}\left(e^{i 2 \alpha}\right)$ invertible, if $\mathcal{M}$ is nondegenerate then

$$
\tilde{r}\left(\tilde{\mathcal{M}}^{*}\right) \cdot \tilde{S}_{\alpha}-\tilde{r}\left(\tilde{\mathcal{M}}^{*}\right) \cdot \tilde{S}_{0}=-\frac{1}{2} \operatorname{Sign} B_{\kappa}\left(e^{i 2 \alpha}\right) .
$$

If $\mathcal{M}$ is degenerate, then after a small perturbation this formula holds.

Proof. The assumption about $B_{\kappa}\left(e^{i 2 \alpha}\right)$ implies that $\mathcal{H}_{A^{\alpha}}^{1}(Y, \partial Y ; s u(2))=0$. By Theorem 3.1 we can find a perturbation $h$ satisfying the hypotheses of Lemma 5.1. This lemma, combined with Corollary 6.2 and Proposition 6.3, proves the theorem. 


\section{References.}

[AM] S. Akbulut and J. McCarthy, Casson's Invariant for oriented homology 3-spheres, an exposition, Mathematical Notes 36, Princeton University Press, 1990.

[F] A. Floer, An instanton invariant for 3-manifolds, Comm. Math. Phys. 118 (1988), 215-240.

[FU] D. Freed and K. Uhlenbeck, Instantons and four-manifolds, Math. Sci. Research Inst. Series, vol. 1, Springer-Verlag, 1984.

[G1] W. Goldman, The symplectic nature of the fundamental groups of surfaces, Adv. Math. 54 (1984), 200-225.

[G2] W. Goldman, Representations of fundamental groups of surfaces, in Proceedings of Special Year in Topology, Maryland 1983-84, Lecture notes in mathematics 1167, Springer-Verlag, 1985.

[H1] C. Herald, Legendrian Cobordism and Chern-Simons Theory on 3Manifolds with Boundary, Comm. Anal. and Geom. 2 (1994), No. 3, 337-413.

[H2] C. Herald, Existence of irreducible representations for knot complements with nonconstant equivariant signature, to appear in Math. Ann.

[H3] C. Herald, Spectral flow, Dehn surgery, and the Casson invariant, preprint.

[He] M. Heusener, $\mathrm{SO}_{3}(\mathbf{R})$-representation curves for two-bridge knot groups, Math. Ann. 298 (1994), 327-348.

[Ho] L. Hörmander, The Analysis of Linear Partial Differential Operators III, Springer-Verlag, 1985.

[JW] L. Jeffrey and J. Weitsman, Bohr-Sommerfeld orbits in the moduli space of flat connections and the Verlinde formula, Comm. Math. Phys. 150 (1992), no. 3, 593-630.

[K] L. Kauffman, On Knots, Annals of Math. Studies 115, Princeton University Press, 1987.

[K] M. Kuranishi, New Proof of the existence of locally complete families of complex structures, Proceedings of the Conference on Complex Analysis, A. Aepplis et al, eds., Springer-Verlag, 1965, 142-154. 
[KKR] P. Kirk, E. Klassen, and D. Ruberman, Splitting the Spectral Flow and the Alexander Matrix, Commentarii Math. Helv. 69 (1994), 375416.

[L] H.B. Lawson, The theory of gauge fields in four dimensions, Reg. Conf. Ser. Math. 58, American Mathematical Society, 1985.

[Li] X.-S. Lin, A knot invariant via representation spaces, J. Diff. Geom. 35 (1992), 337-357.

[MMR] J. Morgan, T. Mrowka, and D. Ruberman, The $L^{2}$-Moduli Space and a Vanishing Theorem for Donaldson Polynomial Invariants, International Press, 1994.

[T] C. Taubes, Casson's invariant and gauge theory, J. Diff. Geom. 31 (1990), 547-599.

[Wa] K. Walker, An Extension of Casson's Invariant, Annals of Mathematics Studies, Study 126, Princeton University Press, 1992.

ReCeived March 14, 1995.

Department of Mathematics and Statistics

SWARTHMORE COLLEGE

500 College Ave.

SWARTHMORE, PA 19081

E-MAIL:CHERALD1@SWARTHMORE.EDU 